\section{(1) bserbations}

ON THE

\section{CASAREAN SECTION AND ON OTHER OBSTETRIC OPERATIONS.}

BY

TIIOMAS RADFORD, M.D.,

F.R.C.P.EDIN, AND F.R.C.S.ENG., ETC.,

HoNokary CONSUlTING PHYSICIAN TO ST. MARY's hospital, MANCHESTER.

\section{Chapter $\mathrm{X}$. \\ Considerations on the Casarean Section as an Operation of Election.}

Is the preceding remarks, I have to my own mind most satisfactorily proved that the Cæsarean section is at least an operation of necessity, and that those measures which have been proposed as substitutes are totally inadequate to supersede it.

The (British) statistics of this operation are most certainly unfavourable; yet it has, I think, been shewn that the great cause of the maternal mortality is avoidable ; and that most of the other alleged causes of this fatality have been pointed out to be subject to control, and that some of them are really preventable, whilst others are remediable.

Although the deaths of the women from this operation, as hitherto performed, are very numerous, yet objections ought not to be raised against it on data so unsatisfactory as those are which now influence the opinions of British practitioners. When I speak of unsatisfactory data, I mean that we should not take an abstract view of them, and attach to them more importance than they deserve. British obstetricians have been guided more from prejudice arising from preconceived opinions, than from an analysis of the real causes of death in these cases.

A comparative estimate of the mortality between this operation and craniotomy has never been fairly made. As regards the Cæsarean section, all the deaths are known, whereas those of craniotomy are only very partially known. This latter operation is not confined to any particular class of cases, but it is performed under very different circumstances and dangers-in some cases of accidents which occur in labours, such as hæmorrhage, convulsion, and other contingent mischief, which happen in women whose constitutional powers are unimpaired; whilst the greater part of the women who have undergone the Cæsarean section have laboured under incurable disease, and have had additional injury inflicted upon them by protraction, and in many cases by the practitioner in his ineffectual performance of craniotomy, etc.

Dr. Joseph Clarke reports 49 cases of craniotomy, in which 16 mothers died, or one in three. Dr. Collins performed craniotomy in 79 cases, in which 15 died, or one in five. Now, in Dr. Clarke's practice collectively, there are recorded 65 deaths;* in Dr. Collins's practice collectively, there are recorded 94 deaths.* 'Thus the statistics of craniotomy, which are indiscriminately made up of all kinds of cases in which it has been performed, show an unfavourable result.

Besides this, we have no account of the injuries which are inflicted on the pelvic organs by the instrument used in this operation.

In 77 cases of Cæasarean section, there were collectively 98 deaths, ${ }^{*}$ the greater portion of which were not due to the operation; but, on the contrary, very many lives might have been saved, if it had been timely and judiciously performed.

Notwithstanding all the preexisting dangers in Cæesarean cases, several recoveries have taken place. These favourable terminations ought to encourage us to hope, and indeed ought to inspire us with confidence, that if the operation were earlier performed, and on a different class of subjects, it would be attended with infinitely more success.

These cases prove that, notwithstanding the serious nature of the constitutional disease which existed in these women, the vital powers were equal to the reparation of both the abdominal and uterine incisions; and also show the fallacy of the opinion that wounds of the uterus are necessarily mortal. The conservative vital powers were wonderfully apparent in the case of Mrs. Sankey, which is related in the London Medical Gazette, also in the Provincial Medical and Surgical Journal. The restorative powers in this individual were really so active as to impress my mind with the conviction that the chance of success would be as great in well conducted Cæsarean cases as that which attends other capital operations.

Another woman (Mrs. Haigh), in whom mollities ossium existed to a great extent, and whose pelvis was very much distorted, showed great restorative powers. She recovered, and lived several years afterwards. She died exhausted by the disease. Post mortem examination revealed no disease in the abdominal or pelvic viscera. The uterine tissue was uniform in appearance, there being no cicatrix to indicate the site of the incision. There was only a single band of lymph, not thicker than a thread, passing from this organ to the peritoneum; so that there existed no mechanical obstacle to the distension and ascent of the uterus, if she had unfortunately become pregnant again; but the moral rule of abstinence prevailed with both her and her husband.

Recoveries after rupture of the uterus afford further evidence that wounds of this organ are not always mortal. The lacerated tissue in these cases must be in a very different, and indeed in a much more unfavourable, condition for uniting, than in Cæsarean cases. In these accidents, the peritoneum, the abdominal and the pelvic viscera, must inevitably sustain very great injury by the escape of the infant, and also very frequently of the placenta, through the uterine rent; and also from the attempts which are made for the delivery of the woman. The same mischief cannot possibly be inflicted by a well conducted Cæsarean operation.

Two instances of recovery after rupture of the uterus have occurred in my practice. One of these women became pregnant several times afterwards. In one of these pregnancies, she went to her full time, and bore a child, which is still alive; and she also aborted several times. During her last labour, and also during the several abortive periods, she had the valuable aid and advice of my respected friend [Continued on page 427.]

* These accounts contain the number of deaths of both women and infants. 
Table of Recorded Cases of Cesarean Sfction in Great Britain and Ireland.

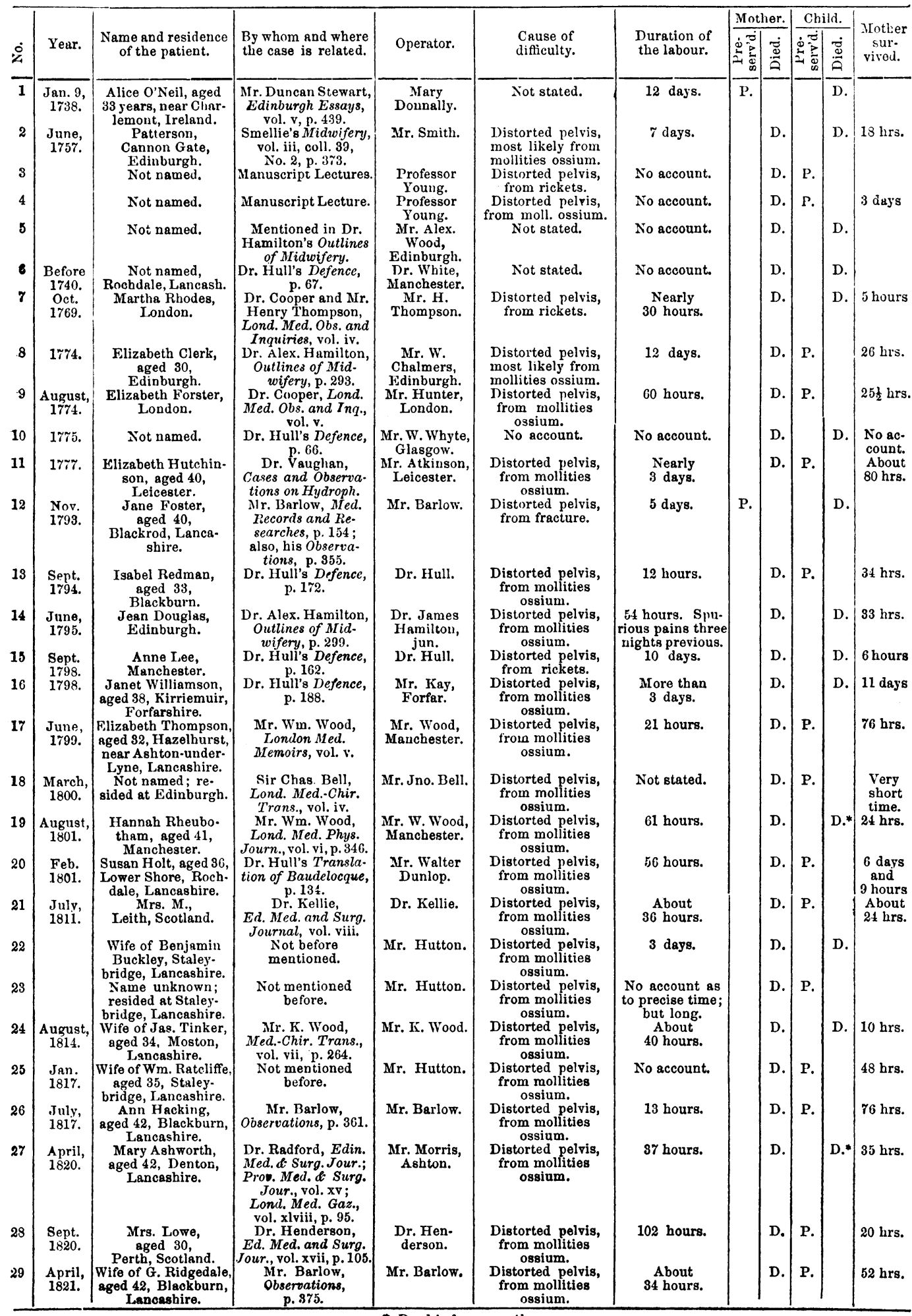

* Dead before operation. 
Tarle of Cases of Cagarean Section (continued).

\begin{tabular}{|c|c|c|c|c|c|c|c|c|c|c|c|}
\hline \multirow[b]{2}{*}{$\dot{0}$} & \multirow[b]{2}{*}{ Year. } & \multirow[b]{2}{*}{$\begin{array}{c}\text { Name and residence } \\
\text { of the patient. }\end{array}$} & \multirow[b]{2}{*}{$\begin{array}{l}\text { By whom and where } \\
\text { the case is related. }\end{array}$} & \multirow[b]{2}{*}{ Operator. } & \multirow[b]{2}{*}{$\begin{array}{l}\text { Cause of } \\
\text { difficulty. }\end{array}$} & \multirow[b]{2}{*}{$\begin{array}{l}\text { Duration of } \\
\text { the labour. }\end{array}$} & \multicolumn{2}{|c|}{ Mnther. } & \multicolumn{2}{|c|}{ Child. } & \multirow[b]{2}{*}{$\begin{array}{l}\text { Mother } \\
\text { sur- } \\
\text { vived. }\end{array}$} \\
\hline & & & & & & & $\begin{array}{ll}0 \\
0 \\
2 & 0 \\
2 & 0 \\
2 & 0 \\
0\end{array}$ & 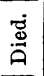 & 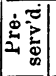 & $\ddot{\otimes}$ & \\
\hline 30 & $\begin{array}{l}\text { May, } \\
1821 .\end{array}$ & $\begin{array}{l}\text { Mary Nixon, } \\
\text { aged } 39, \\
\text { Manchester. }\end{array}$ & $\begin{array}{l}\text { Dr. Radford, Edin. } \\
\text { Med.E Surg.Jour.; } \\
\text { Lond. Med. Gaz., } \\
\text { vol. xlvii, p. 98; } \\
\text { Prov. Med. \& Sur.Jl., }\end{array}$ & Mr. Wilson. & $\begin{array}{l}\text { Distorted pelvis, } \\
\text { from mollities } \\
\text { ossium. }\end{array}$ & 22 bours. & & D. & & D. & $67 \frac{1}{2} \mathrm{hrs}$. \\
\hline 31 & $\begin{array}{l}\text { April } \\
1826 .\end{array}$ & $\begin{array}{l}\text { M. R., aged 22, } \\
\text { Stobsmuir, } \\
\text { Scotland. }\end{array}$ & $\begin{array}{c}\text { Mr. Crichton, } \\
\text { Mundee, Ed. Med. } \\
\text { and Surg. Journ., } \\
\text { vol. xxx, p. } 53 \text {. }\end{array}$ & Mr. Crichton. & $\begin{array}{l}\text { Distorted pelvis, } \\
\text { from fracture, etc. }\end{array}$ & 6 days. & & D. & P. & & 8 hours. \\
\hline 32 & $\begin{array}{c}\text { August, } \\
1826 .\end{array}$ & $\begin{array}{l}\text { Mary Forrest, aged } \\
35, \text { three miles from } \\
\text { Blackburn, Lancash. }\end{array}$ & $\begin{array}{c}\text { Mr. Barlow, } \\
\text { Lond. Med. \& Surg. } \\
\text { Jour., vol. iv. }\end{array}$ & Mr. Barlow. & $\begin{array}{c}\text { Distorted pelvis, } \\
\text { from mollities } \\
\text { ossium. }\end{array}$ & $\begin{array}{c}\text { From } 30 \text { to } 36 \\
\text { hours. }\end{array}$ & & D. & P. & & $\begin{array}{l}\text { More } \\
\text { than } \\
3 \text { days. }\end{array}$ \\
\hline 33 & $\begin{array}{l}\text { Sept. } \\
\text { 1829. }\end{array}$ & $\begin{array}{l}\text { Irs. MI., aged 26, } \\
\text { Belfast. }\end{array}$ & $\begin{array}{c}\text { Communicated by } \\
\text { Dr. W. Camphell, } \\
\text { Edin. Med.\& Surg. } \\
\text { Jour., v. xxxv, p. } 351 .\end{array}$ & Dr. M'Kibbin. & $\begin{array}{l}\text { Distorted pelvis, } \\
\text { from a large exosto- } \\
\text { sis arising from } \\
\text { the sacrum. }\end{array}$ & About 30 hours. & & D. & & D.t & $17 \mathrm{hrs}$. \\
\hline 34 & $\begin{array}{l}\text { Nov. } \\
1834 .\end{array}$ & $\begin{array}{l}\text { Mrs. - } \\
\text { Dubliu. }\end{array}$ & $\begin{array}{c}\text { Dr. Montgomery, } \\
\text { Dublin Med.Journ., } \\
\text { vol. vi. }\end{array}$ & Mr. Porter. & $\begin{array}{l}\text { Fibrous tumour, } \\
\text { growing from sub- } \\
\text { stance of uterus } \\
\text { and covered by } \\
\text { peritoneum. }\end{array}$ & 18 to 20 hours. & & D. & & D.t & $21 \frac{1}{2} \mathrm{hrs}$. \\
\hline 35 & $\begin{array}{l}\text { April, } \\
1334 .\end{array}$ & $\begin{array}{c}\text { Mary Bamford, } \\
\text { aged 38, Great } \\
\text { Easton, Rockingham. }\end{array}$ & $\begin{array}{c}\mathrm{Mr} \text {. T. I. Greaves, } \\
\text { Lancet, vol. ii, 1833-4. }\end{array}$ & Mr. Greaves. & $\begin{array}{l}\text { Distorted pelvis, } \\
\text { from mollities } \\
\text { ossium. }\end{array}$ & About 34 hours. & P. & & P. & & \\
\hline 36 & $\begin{array}{l}\text { May, } \\
1835 .\end{array}$ & $\begin{array}{c}\text { Sarah Bute, } \\
\text { aged } 36, \\
\text { Birmingham. }\end{array}$ & $\begin{array}{c}\text { Mr. Knowles, } \\
\text { Trans. Prov. Med. } \\
\text { Assoc., vol. iv, p. } 376 .\end{array}$ & Mr. Knowles. & $\begin{array}{l}\text { Distorted pelvis, } \\
\text { from mollities } \\
\text { ossium. }\end{array}$ & About 30 hours. & P. & & P. & & \\
\hline 37 & $\underset{18.10 .}{\operatorname{sug} .14}$ & $\begin{array}{l}\text { Mary Ann Joues, } \\
\text { aged } 39 \\
\text { Manchester. }\end{array}$ & $\begin{array}{l}\text { Mr. Jas. Whitehead, } \\
\text { Manchester, } \\
\text { Merl. Gaz., vol. xxvii, } \\
\text { p. } 939,1840.41 .\end{array}$ & $\begin{array}{l}\text { Mr. White. } \\
\text { head. }\end{array}$ & $\begin{array}{l}\text { Distorted pelvis, } \\
\text { from mollities } \\
\text { ossium. }\end{array}$ & $\begin{array}{l}24 \text { hours; in } \\
\text { active labour } \\
\text { for } 2 \text { to } 3 \text { bours. }\end{array}$ & P. & & P. & & $\begin{array}{l}32 \text { days } \\
10 \text { hrs. }\end{array}$ \\
\hline 38 & & $\begin{array}{l}\text { Name not given, or } \\
\text { age; private note, } \\
\text { aged } 30 .\end{array}$ & $\begin{array}{l}\text { Mr. Dendy, Medical } \\
\text { Society of London, } \\
\text { Lancet, } 1842-43, \\
\text { vol. xliii, p. } 691 .\end{array}$ & $\begin{array}{l}\text { Mr. Bryant, } \\
\text { Lambeth. }\end{array}$ & $\begin{array}{l}\text { Distorted pelvis, } \\
\text { by rickets. }\end{array}$ & 24 hours. & & D. & P. & & $60 \mathrm{hrs}$. \\
\hline 39 & $\begin{array}{c}\text { Oct. 1\% } \\
1842 .\end{array}$ & $\begin{array}{l}\text { Mary Davis, } \\
\text { aged 23, } \\
\text { Reading. }\end{array}$ & $\begin{array}{c}\text { Mr. T. B. Hooper, } \\
\text { Reading Medical } \\
\text { Society, Lancet, 1843, } \\
\text { vol. xliii, p. } 689 .\end{array}$ & Mr. Hooper. & $\begin{array}{l}\text { A large tumour, } \\
\text { arising from the } \\
\text { sacrum. }\end{array}$ & 3 days. & & D. & & D.t & $40 \mathrm{hrs}$. \\
\hline 40 & $\begin{array}{c}\text { Mar. } 8 \\
1842 .\end{array}$ & $\begin{array}{l}\text { Mary Jepson, } \\
\text { aged 43, } \\
\text { a weaver, } \\
\text { Darwen. }\end{array}$ & $\begin{array}{c}\text { Mr. S. H. Wraith, } \\
\text { Darwen, Prov. Med. } \\
\text { Jour., vol. v, p. } 329, \\
1842-43 .\end{array}$ & Mr. Wraith. & $\begin{array}{l}\text { Distorted pelvis, } \\
\text { from malacosteon. }\end{array}$ & 10 hours. & & D. & & D. & 3 hours. \\
\hline 41 & $\begin{array}{l}\text { Feb. } 22, \\
1843 .\end{array}$ & $\begin{array}{c}\text { Nary Forrest, } \\
\text { aged 3:, a weaver, } \\
\text { Stockport. }\end{array}$ & $\begin{array}{c}\text { Dr. Hadford, } \\
\text { Med..Gazette, } \\
\text { vol. xlvii, p. 801 ; } \\
\text { Prov. Med.\& Sur. Jl., } \\
\text { vol. xv, p. 28\%, 1851. }\end{array}$ & Dr. Radford. & $\begin{array}{l}\text { Distorted pelvis, } \\
\text { from malacosteon. }\end{array}$ & 53 hours. & & D. & & D. & $\begin{array}{l}\text { About } \\
27 \mathrm{hrs} \text {. }\end{array}$ \\
\hline 42 & $\begin{array}{l}\text { Tnly, } \\
10: 55 .\end{array}$ & $\begin{array}{l}\text { Betty Wilcock, } \\
\text { aged nearly } 49 .\end{array}$ & $\begin{array}{c}\text { Messrs. Hardy and } \\
\text { Bailey, Ass. Med.Jl., } \\
\text { vol.iv, p. } 45,1856 .\end{array}$ & Mr. Bailey. & $\begin{array}{l}\text { Distorted pelvis, } \\
\text { from mollities } \\
\text { ossium. }\end{array}$ & 83 hours. & & D. & P.* & & $61 \frac{1}{2}$ hrs. \\
\hline 43 & $\begin{array}{c}\text { Oct. 18, } \\
1837 .\end{array}$ & $\begin{array}{l}\text { E. Hull, aged 25, } \\
\text { Sunderland. }\end{array}$ & $\begin{array}{l}\text { Mr. J. Ward, } \\
\text { Lond. Med. Gazette, } \\
\text { vol. xxi, p. } 817 .\end{array}$ & Mr. J. Ward. & $\begin{array}{l}\text { Distorted pelvis, } \\
\text { from (presumed) } \\
\text { rickets. }\end{array}$ & 27 to 30 hours. & & D. & & D.t & $\begin{array}{l}5 \text { days } \\
7 \frac{1}{2} \text { brs. }\end{array}$ \\
\hline 44 & 1840. & .. & $\begin{array}{c}\text { Dr. Churchill's } \\
\text { Operative Midwifery, } \\
\text { Table, p. 205, com- } \\
\text { municated in a letter } \\
\text { to Dr. C. }\end{array}$ & $\begin{array}{l}\text { Dr. Elliott, } \\
\text { Waterford, } \\
\text { Ireland. }\end{array}$ & $\begin{array}{l}\text { Distortion of pelvis; } \\
\text { kind not } \\
\text { mentioned. }\end{array}$ & & & D. & & D. & \\
\hline 45 & $\begin{array}{l}\text { Feb. } \\
1 \otimes 12 .\end{array}$ & $\begin{array}{c}\text { Helen McKenzie, } \\
\text { aged } 3 J .\end{array}$ & $\begin{array}{l}\text { Mr. Alex. Ross, } \\
\text { London and Edin. } \\
\text { Monthly Journal, } \\
\text { vol. ii, p. } 425 .\end{array}$ & $\begin{array}{l}\text { Mr. A. Ross, } \\
\text { Invergordon. }\end{array}$ & $\begin{array}{l}\text { Distortion of pelvis, } \\
\text { most probably mol- } \\
\text { lities ossium (outlet } \\
\text { contracted). Tuber- } \\
\text { osities of ischia ap- } \\
\text { proximated very } \\
\text { closely; coccyx clos- } \\
\text { ing up lower part. }\end{array}$ & $\begin{array}{c}\text { Midwife stated } \\
\text { she had been in } \\
\text { labour, more or } \\
\text { less, for 12 days. } \\
\text { Much time elap. } \\
\text { sed after Mr. } \\
\text { Ross decided on } \\
\text { necessity of } \\
\text { operation. }\end{array}$ & & D. & P. & & $\begin{array}{l}5 \text { days } \\
7 \text { hrs. }\end{array}$ \\
\hline 46 & $\begin{array}{c}\text { August, } \\
18.4 .\end{array}$ & $\begin{array}{l}\text { Rebecca Brooks, } \\
\text { aged } 27, \\
\text { Welford. }\end{array}$ & $\begin{array}{l}\text { Mr. Fred. Cox, } \\
\text { Prov. Aled. and } \\
\text { Surg. Journal, } \\
\text { vol. viii, p. } 382 .\end{array}$ & $\begin{array}{l}\text { Mr. F. Cox, } \\
\text { Welford. }\end{array}$ & $\begin{array}{c}\text { Distortion of pelvis, } \\
\text { most likely rickety, } \\
\text { but not stated. }\end{array}$ & 80 to 40 hours. & & D. & & D. $\ddagger$ & 54 hrs. \\
\hline 47 & $\begin{array}{c}\text { Feb. 21, } \\
1 \times 15 .\end{array}$ & $\begin{array}{l}\text { Wife of Richard } \\
\text { Instan, aged } 40 .\end{array}$ & $\begin{array}{c}\text { Mr.J. Milman Coley, } \\
\text { Pamphlet. }\end{array}$ & Mr. Coley. & $\begin{array}{l}\text { Distortion of pelvis, } \\
\text { said to be rickety. }\end{array}$ & At least 10 days. & & D. & & D. & \\
\hline 48 & $\begin{array}{c}\text { March, } \\
1815 .\end{array}$ & $\begin{array}{c}\text { Mrs. R., } \\
\text { Shettlestone. }\end{array}$ & $\begin{array}{l}\text { Mr. William Lyon, } \\
\text { Lon. \& Edin. Jour. } \\
\text { of Med. Science, } \\
\text { vol. v, p. } 885 .\end{array}$ & Mr. Lyon. & $\begin{array}{l}\text { A large tumour, the } \\
\text { size of a child's } \\
\text { head. }\end{array}$ & 72 hours. & & D. & P. & & $36 \mathrm{hrs.}$ \\
\hline 49 & $\begin{array}{l}\text { Nov. } \\
1845 .\end{array}$ & $\begin{array}{l}\text { Mrs. Sankey, } \\
\text { aged 41, } \\
\text { Salford. }\end{array}$ & $\begin{array}{c}\text { Dr. Radford, } \\
\text { Lond. MIed. Gaz., } \\
\text { vol. xlvii, p. } 894 ; \\
\text { Prov. Med.\& Sur.Jl., } \\
\text { vol. xv, p. } 315,1851 .\end{array}$ & $\begin{array}{c}\text { Mr. Goodman, } \\
\text { Assisted by } \\
\text { Dr. Radford. }\end{array}$ & $\begin{array}{l}\text { Distortion of pelvis, } \\
\text { from mollities } \\
\text { ossium. }\end{array}$ & 12 to 14 hours. & P. & & $\mathbf{P}$. & & Lived. \\
\hline 50 & $\begin{array}{l}\text { Jan. } \\
184 \pi .\end{array}$ & $\begin{array}{c}\text { Sarah Bartlett, aged } \\
3 \%, \text { removed for oper- } \\
\text { ation to St. Bartho- } \\
\text { lomew's Hospital, } \\
\text { London. }\end{array}$ & $\begin{array}{l}\text { Lancet, } 1847 \\
\text { vol. } \mathrm{i}, \mathrm{p}, 139\end{array}$ & $\begin{array}{l}\text { Mr. Skey, } \\
\text { London. }\end{array}$ & $\begin{array}{l}\text { Distortion, } \\
\text { from rickets. }\end{array}$ & 5 hrs. 5 min. & & D. & P. & & 36 hrs. \\
\hline
\end{tabular}




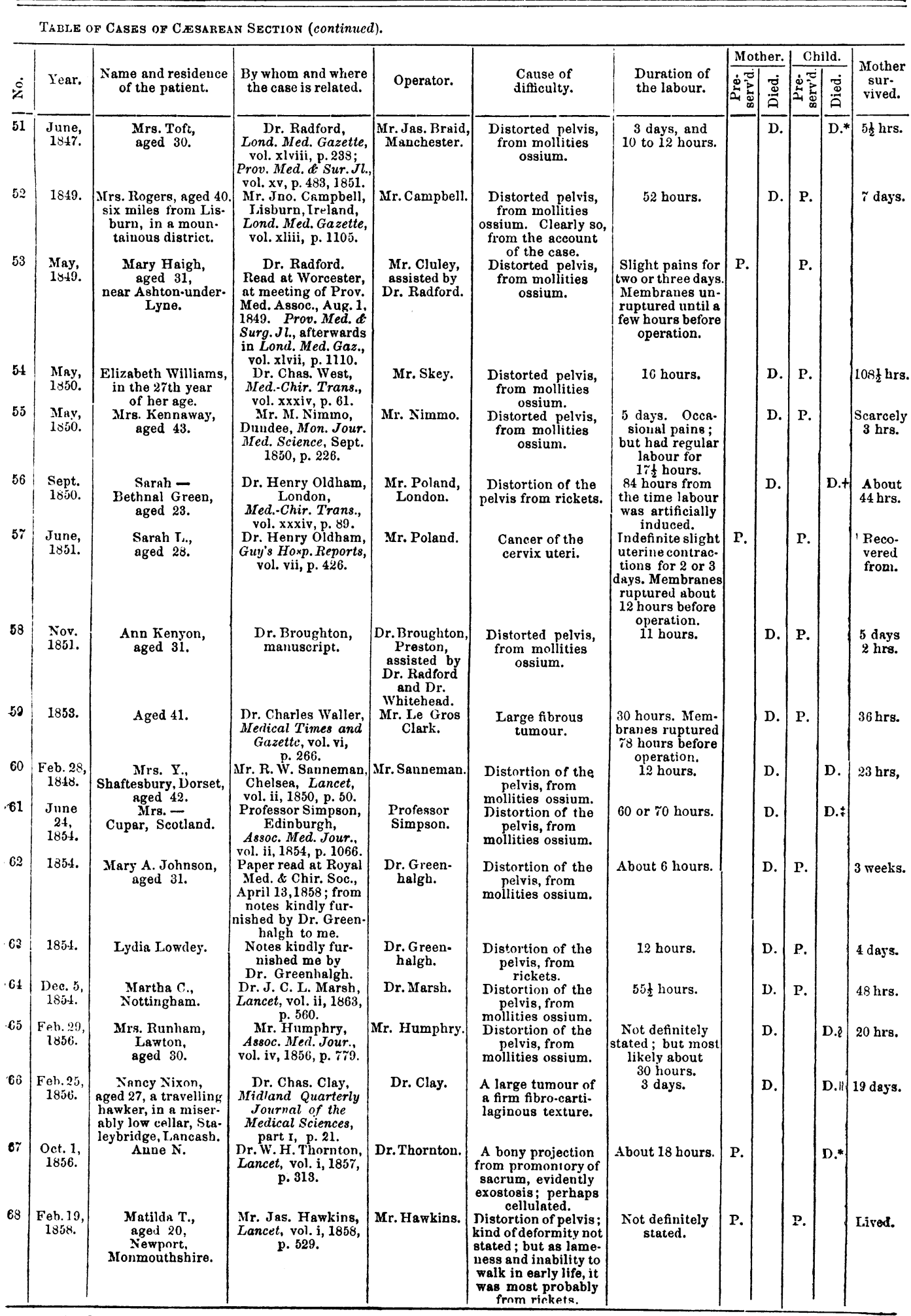

* Dead before operation.

Embryotomy had begun; the arm remored.
+ Head perforated.

a Alive when extracted; but died in a few seconds.
‡ Living; died soon after. 426 
Table of Cases of Casaraan Section (concluded).

\begin{tabular}{|c|c|c|c|c|c|c|c|c|c|c|c|}
\hline \multirow[b]{2}{*}{$\dot{\circ}$} & \multirow[b]{2}{*}{ Year. } & \multirow[b]{2}{*}{$\begin{array}{c}\text { Name and residence } \\
\text { of the patient. }\end{array}$} & \multirow[b]{2}{*}{$\begin{array}{l}\text { By whom and where } \\
\text { the case is reluted. }\end{array}$} & \multirow[b]{2}{*}{ Operator. } & \multirow[b]{2}{*}{$\begin{array}{l}\text { Canse of } \\
\text { difficulty. }\end{array}$} & \multirow[b]{2}{*}{$\begin{array}{l}\text { Duration of } \\
\text { the labour. }\end{array}$} & \multicolumn{2}{|c|}{ Mother. } & \multicolumn{2}{|c|}{ Child. } & \multirow[b]{2}{*}{$\begin{array}{l}\text { Mother } \\
\text { sur: } \\
\text { vived. }\end{array}$} \\
\hline & & & & & & & 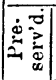 & $\underset{\Phi}{\stackrel{\oplus}{0}}$ & 定: & \begin{tabular}{l}
$\stackrel{\overbrace{}}{0}$ \\
\hdashline
\end{tabular} & \\
\hline 69 & $\begin{array}{c}\text { July 12, } \\
1858 .\end{array}$ & $\begin{array}{c}\text { Mrs. N., aged } 30, \\
\text { I Iarrington; brought } \\
\text { to University Col- } \\
\text { lege Hospitul. }\end{array}$ & $\begin{array}{l}\text { Dr. Murphy, } \\
\text { Dub. Quar. Jour. of } \\
\text { Med. Sc., vol. xxvii, } \\
\text { (new series), p. 108. }\end{array}$ & Mr. Quain. & $\begin{array}{l}\text { Distortion of the } \\
\text { pelvis, from } \\
\text { mollities ossium. }\end{array}$ & $\begin{array}{l}\text { As far as can be } \\
\text { computed, } \\
4 \text { days. }\end{array}$ & & D. & & D. & $\begin{array}{l}\text { Nearly } \\
48 \text { hrs. }\end{array}$ \\
\hline 70 & $\begin{array}{l}\text { Dec. } \\
1859 .\end{array}$ & $\begin{array}{l}\text { Mrs. H., Walton-le- } \\
\text { dale, near Preston, } \\
\quad \text { Lancashire. }\end{array}$ & $\begin{array}{c}\text { Dr. H. Ashton, } \\
\text { Lancet, vol. i, 1860, } \\
\text { p. } 4 \dot{4} 0 .\end{array}$ & Dr. Ashton. & $\begin{array}{l}\text { Distortion of the } \\
\text { pelvis, from } \\
\text { mollities ossium. }\end{array}$ & $\begin{array}{c}\text { As far as can be } \\
\text { ascertained from } \\
\text { the data, about } \\
17 \text { or } 18 \text { hours. }\end{array}$ & & D. & P. & & $25 \mathrm{hrs}$. \\
\hline 71 & $\begin{array}{c}\text { Dec. 10, } \\
1860 .\end{array}$ & Emma P. & $\begin{array}{l}\text { Dr. Jas. Edmunds, } \\
\text { Lancet, vol. i, 1861, } \\
\text { p. } 4 .\end{array}$ & Dr. Edmunds. & $\begin{array}{l}\text { Hard cancer of os } \\
\text { aud cervix uteri. }\end{array}$ & Fully 6 days. & P. & & P. & & Lived. \\
\hline 72 & $\begin{array}{c}\text { Feb. 2, } \\
1861 .\end{array}$ & $\begin{array}{l}\text { Isabella King, un. } \\
\text { married, aged } 23 \text {, } \\
\text { Aberdeen, Scotland. }\end{array}$ & $\begin{array}{l}\text { Jr. Robert Dyce, } \\
\text { Edin. MIed. Jour., } \\
\text { vol. vii, p. } 895 .\end{array}$ & Dr. Dyce. & $\begin{array}{l}\text { Distortion of the } \\
\text { pelvis, from } \\
\text { rickets. }\end{array}$ & $\begin{array}{l}\text { As far as can be } \\
\text { computed, } \\
4 \text { to } 5 \text { days. }\end{array}$ & & D. & & D.* & $43 \mathrm{hrs.}$ \\
\hline$\% 3$ & $\begin{array}{c}\text { Aug. } 7 \\
1862 .\end{array}$ & $\begin{array}{c}\text { E. M., } \\
\text { unmarried, } \\
\text { aged 17, } \\
\text { Tottenhall. }\end{array}$ & $\begin{array}{l}\text { Dr. David Jolunson, } \\
\text { Lancet, vol. ii, 1862, } \\
\text { p. } 475 .\end{array}$ & Dr. Juhnson. & $\begin{array}{l}\text { Small underdeve- } \\
\text { loped pelvis, perhaps } \\
\text { rickety. Antero-pos- } \\
\text { terior diameter only, } \\
\text { ascertained pcr } \\
\text { vaginam to be } 2 \frac{1}{2} \text { in. } \\
\text { Preteruatural } \\
\text { position of child, } \\
\text { right hand and the } \\
\text { two feet. }\end{array}$ & $\begin{array}{l}12 \text { hours, when } \\
\text { first seen by Dr. } \\
\text { J.; afterwards } \\
\text { the time in his } \\
\text { attempt to deli. } \\
\text { ver, etc., } 17 \mathrm{hrs} \text {. }\end{array}$ & & D. & & D.t & $46 \mathrm{hrs}$. \\
\hline$\pi 1$ & $\begin{array}{c}\text { Dec. 24, } \\
1862 .\end{array}$ & $\begin{array}{c}\text { Mary Ann - } \\
\text { unmarried, } \\
\text { aged } 42, \\
\text { Kingswood, Bristol. }\end{array}$ & $\begin{array}{c}\text { Dr. J. G. Swayne, } \\
\text { Obstetric Trans., } \\
\text { vol. v, 1863, p. } 84 .\end{array}$ & $\begin{array}{l}\text { Mr. Coe, } \\
\text { at the } \\
\text { Bristol } \\
\text { General } \\
\text { Hospital. }\end{array}$ & $\begin{array}{l}\text { Distortion of the } \\
\text { pelvis, from con- } \\
\text { genital malforms- } \\
\text { tion. It was like that } \\
\text { produced by rickets. }\end{array}$ & $\begin{array}{l}60 \text { hours befor } \\
\text { she came to the } \\
\text { hospital, and a } \\
\text { few hours after } \\
\text { her arrival. }\end{array}$ & & D. & P. & & $42 \mathrm{hrs}$. \\
\hline$\tau 5$ & 1863. & $\begin{array}{l}\text { Eliza Hubbard, } \\
\text { St. Bartholomew's } \\
\text { Hospital. }\end{array}$ & $\begin{array}{l}\text { Dr. Greenhalgh, } \\
\text { private } \\
\text { communication. }\end{array}$ & Mr. Skey. & Medullary tumour. & 18 hours. & & D. & & D. & $18 \mathrm{hrs}$. \\
\hline$\% 6$ & $\begin{array}{c}\text { Sep. 10, } \\
\text { Iธ61. }\end{array}$ & $\begin{array}{l}\text { Mary Salimson, } \\
\text { City of Iondon } \\
\text { Lying-in Hospital, } \\
\text { aged } 28 .\end{array}$ & $\begin{array}{c}\text { Dr. Greenhalgh, } \\
\text { private } \\
\text { communication. }\end{array}$ & $\begin{array}{l}\text { Dr. Green- } \\
\text { halgh. }\end{array}$ & $\begin{array}{l}\text { Distortion of the } \\
\text { pelvis, from } \\
\text { rickets. }\end{array}$ & About 41 hours. & & D. & P. & & $48 \frac{1}{2} \mathrm{hrs}$. \\
\hline$\pi$ & $\begin{array}{c}\text { Nov. } 23 \\
1864 .\end{array}$ & $\begin{array}{c}\text { Ann Burgess, Xo. 1, } \\
\text { Brown Street, Acton } \\
\text { Street, London Koad, } \\
\text { Manchester. }\end{array}$ & Dr. Thomas Pigg. & Dr. Clay. & Malacosteon. & $\begin{array}{l}\text { Strong pains for } \\
24 \text { hours, but } \\
\text { had probably } \\
\text { been in labour } \\
\text { to some extent } \\
\text { before this. }\end{array}$ & & D. & P. & & 3 das 8. \\
\hline
\end{tabular}

* Turning ineffectually attempted. Craniotomy was also unsuccessfully performed.

+ Dead before operation.

Mr. Hunt. Many years afterwards, she died in the Manchester Workhouse. Her body was inspected by Mr. Ilunt, in the presence of Dr. Francis and of myself. There was not the slightest trace of the cicatrix in the womb to be seen; but there was a band of slight adhesion to the ilium.

I was consulted by Dr. Clay in his first case of large ovarian tumour, and attended along with him both before, during, and after the operation. I take this opportunity of saying I consider the successful issue of this operation as the commencement of a new era in the history of ovariotomy. It had not been attempted for many years before; and, at the time of its performance, it did not stand as if it were a recognised surgical operation. I attended also along with Dr. Clay many of his next succeeding cases, being present at all the operations. I took great interest in these cases, not only on account of that which necessarily belonged to them, but also because the results analogically tended to substantiate my views relative to the probable success of the Cæsarean section. It is nevertheless true, that the influence in these two classes of cases is not quite the same; yet there is, however, sufficient similarity between them to lead us to trust more in abdominal surgery. In ovariotomy, there is certainly no uterine incision; but there is a necessary division to be made of the connecting tissue which exists between the tumour and the uterus. In many of these cases, extensive adhesions, which exist between the tumour and the peritoneum, etc., have to be separated. It is, however, evident that during the progressive development of an ovarian tumour, the sympathy of the peritoneum, etc., must in some measure be blunted, and consequently its susceptibility to mechanical injury must be diminished.

'The rest of Dr. Clay's successful cases, and likewise those of Mr. Spencer Wells, and all those which have occurred to other practitioners, collectively afford strong evidence of the safety of abdominal incision.

The operations performed by my esteemed friend Dr. Blundell on animals, to prove some important physiological facts, likewise afford substantial evidence that abdominal wounds are very much safer than has been usually considered.

In my introductory remarks, I stated that I should not bring forward any statistical data, as shown from the result of foreign cases of the Cæsarean section, although I feel quite sure that the comparative position of this operation has been damaged by the omission. Continental success in this operation has been remarkably great, when compared with the results of British practice. There have been many instances of two or three successful operations on the same woman.

Having, as I sincerely hope, faithfully and candidly placed all the circumstances appertaining to the two operations-the Cæsarean section and craniotomy-before the profession, it now only remains for me to bring forward my proposition, first made in 1843 . Deep reflection since that period, and a 
strong sense of humanity, have induced me further to declare that the Crasarean section should be generally performed as an operation of election; and that craniotomy should be as far as possible abolished, and ought only to be performed as an operation of necessity, except (as already adverted to) in a very few cases.* I am quite aware that many of the opinions I have so urgently stated in the foregoing remarks are at variance with those of the profession generally; yet they have been most conscientiously advocated. They originated from the dictates of humanity, to try to extinguish as far as possible that dreadful expedient-nay, shall I not call it murderous operation?-craniotomy.

Having now fully and without the least reserve put my views into the hands of the profession, I consider I am only doing justice to myself in declaring that I shall not feel called upon to enter into any controversial defence of them.

\section{Ooriginal Commaniations.}

\section{ON PUERPERAL FEVER.}

By T. Sxow BECK, M.D.Lond., F.R.S., Member of the Royal College of Physicians, London.

[Read before the Obstetrical Society of London, February 1st, 1865.]

IN July 1860, I attended Mrs. B. in her first confinement. She had been married about twelve months, was 24 years of age, of rather short stature and good conformation, had always enjoyed good health, and during her pregnancy "never suffered any inconvenience",

On July 24th, she complained of frequent pains in the stomach, which prevented her procuring rest; but without any vaginal discharge. The orifice of the uterus was soft and open, so as to admit the end of the finger.

The following day, the pains were more frequent and of longer duration, became more distressing to her, and were complained of as of a forcing character, chiefly seated in the bottom of the back-the sacrum. The orifice of the uterus was dilated to the size of a five-shilling piece; the membranes entire. There was no vaginal discharge.

The pains continued much the same during the night, but became more decided, retaining their former character, on the afternoon of the 26th. The head descended into the pelvis, and remained stationary for some hours. Ergot of rye and borax were administered without advantage. She became niuch fatigued. The small forceps vas introduced, and a small living female child readily extracted, without any perceptible laceration of the perinæum.

Ergot of rye and borax were again given, but without producing any perceptible effect. The placenta was removed by gentle traction on the cord, combined with pressure on the abdomen. Some hæmorrhage followed; and, after it had continued about an hour, I introduced the hand, and found a large, flabby, open uterus, which could not be distinguished through the walls of the abdomen, and which remained inert under the combined influence of the hand internally and pressure externally on the abdomen. Ice was now passed up into the orifice of the uterus, when a slow and languid contraction followed-sufficient to prevent further hæmorrlage, but not sufficient to induce a firm contracted organ. I

* See remarks, page 344 . reluctantly desisted from further efforts in this direction, by reason of the repeated solicitations of her friends.

She passed a good night; and the succeeding daythe 27 th-was so well that she considered "keeping in bed was only a farce".

On the 28th, I was struck with the alteration in her expression, which was anxious and somewhat pinched. The complexion bore a thick muddy hue; she complained of feeling inwardly cold, but had no decided shivering; the pulse was 110 , soft, and otherwise natural ; the skin cool, and moistened with perspiration, which had a peculiar acrid odour. She complained of pain in the left lumbar region, which extended round the abdomen in a line with the crest of the pubic bone, and induced considerable difficulty in turning in bed. The walls of the abdomen were tender in these situations; the lochia not much in amount, and rather offensive; no milk had appeared in the breasts ; there were no after-pains ; no vomiting; the bowels had not been relieved. A dose of calomel and opium was given, and followed by a gentle saline aperient.

July 29th. A strong, faint, and peculiar offensive odour pervaded the room. The skin was soft, and freely perspiring, which imbued the night-dress with moisture, whilst the exhalation from it appeared to hang about the bed. The expression was anxious and depressed; no pain in the head; mental faculties appeared perfect; no delirium ; feeling of great exhaustion; tongue clean and red; not much thirst; she had occasionally vomited a little froth, slightly tinged with yellow; the bowels were moved several times, the motions being liquid and very offensive; the urine was apparently natural; pulse from 120 to 130, small, and easily compressed. The pain in the side was relieved, and she could turn in bed with more ease; but a more general soreness existed over the whole abdomen on deep pressure, particularly around the navel and over the hypogastrium. The walls of the abdomen were also tender to gentle pressure. The calomel and opium were continued every four hours, combined with a stimulating diaphoretic mixture, and turpentine fomentations were applied to the abdomen.

July 30th. The odour in the room had nearly disappeared; the offensive odour of the lochia was also gone. She expressed herself as being better. The expression of the face was improved, but still oppressed; and the complexion muddy. The perspiration was much diminished; the pulse from 130 to 140 , weak, and very easily compressed; the tongue clean; no great thirst. She continued to vomit a little froth occasionally; did not complain of pain in the abdomen, unless under firm pressure, when the tenderness was about the same. The walls of the abdomen were more prominent and tympanitic on percussion. The mental faculties were unimpaired.

In the evening, I met Dr. Murphy in consultation, who took a most unfavourable view of the case, although the symptoms continued slightly improved. Camphor and opium, in pills, were directed to be substituted for the former pills; and the other remedies to be continued.

On the following morning-July 31st-she was evidently sinking. In the night, she had vomited some coffee-grounds looking matter upon the pillow, and had slightly wandered in her sleep; was lying on the left side; breathing sighing; could not be roused to consciousness; vomited occasionally on the pillow; the pulse very rapid, and fluttering.

She gradually sank, and died about noon.

The vomited matters, under the microscope, showed many fat-globules and portions of muscular fibre. The black points were composed of a congeries of 